\title{
Chapter 19 \\ For the Learning of Mathematics: \\ An Introduction to the Journal \\ and the Writing Within It
}

\author{
Richard Barwell and David A. Reid
}

\begin{abstract}
This chapter provides an overview of the journal For the Learning of Mathematics (FLM) from the point of view of its current editor and his immediate predecessor. It begins with a brief description of the nature of genres, especially in academic writing and more specifically in mathematics education. The aims of the journal and how these are situated in its history are described. The particular genres of writing in FLM are then illustrated with examples. The technicalities of submitting articles to FLM are outlined.
\end{abstract}

Keywords For the Learning of Mathematics - Genre • Academic writing • Mathematics education journals

\subsection{Introduction}

What is distinctive about the journal For the Learning of Mathematics (FLM)? As editors of the journal (past and present), we both believe the writing in FLM to be distinctive, in the sense of having unique style and purpose amongst journals in our field, but how so? And why? In this overview of FLM, we take a genre perspective, with which we first discuss academic publishing in mathematics education in general, and then discuss the particular nature of FLM. We write as current editor (DAR) and previous editor (RB), and we have both been associated with FLM for many years as board members, reviewers and associate editors, prior to our editorial terms.

In research on academic writing and academic literacy, the concept of genre is widely used to examine and understand differences in the organisation, style and

\author{
R. Barwell \\ University of Ottawa, Ottawa, Canada \\ e-mail: richard.barwell@uottawa.ca \\ D. A. Reid ( $\square)$ \\ University of Bremen, Bremen, Germany \\ e-mail: dreid@uni-bremen.de \\ (C) The Author(s) 2019 \\ G. Kaiser and N. Presmeg (eds.), Compendium for Early Career \\ Researchers in Mathematics Education, ICME-13 Monographs, \\ https://doi.org/10.1007/978-3-030-15636-7_19
}


purpose of different kinds of text (see, for example, Swales 1990). This notion is therefore a useful way to understand similarities and differences in the kinds of writing that appear in academic journals in mathematics education. We begin with a brief description of the nature of genres, especially in academic writing and more specifically in mathematics education. We then describe the aims of FLM, and situate these aims in its history. Turning to examples of articles published in FLM over the years, we discuss some of the genres of writing found in FLM and qualities that make articles appropriate for the journal. Finally, we outline the technicalities of submitting articles to FLM.

\subsection{Genre}

In linguistics the term genre refers, roughly, to recognizable textual forms or structures. Everyday examples of textual genres include recipes, instruction manuals, crosswords, or food labels. There are many perspectives on genre and many approaches to genre analysis, ranging from formalist perspectives, in which genres are defined entirely by their structural features, to social perspectives that understand genres as culturally situated activities. As a starting point for this chapter, we recognize that genres have both recognizable textual features and communicative functions. For example, recipes generally include a sequence of often numbered instructions (a textual feature) and are designed to guide readers through a process to produce some kind of dish, such as a cake.

Swales (e.g., 1990) has conducted significant research on genres of academic text. His treatment of genre develops our basic definition. We summarise some key ideas, and illustrate them with reference to the common mathematics classroom genre of word problems, since this genre is familiar to all mathematics educators. We then apply these ideas to academic journal writing.

Swales underlines the relationship between genres and what he calls discourse communities. That is, genres are recognizable to groups of people who interact around some common activity or purpose (see Swales 1990, p. 58). A word problem is instantly recognizable to any mathematics teacher, as well as to their students. Word problems have a common structure (Gerofsky 1996), and a particular purpose: to rehearse the application of some previously taught aspect of school mathematics to a situation described in the problem. Both the structure and the purpose are recognizable to people who work regularly with word problems.

The recognizable nature of genres within a discourse community is linked to a broader cultural function of genres: they are a link between past and present (Swales 1990, p. 45). Genres are 'handed down' from one generation to the next, providing stability within cultures over time and, thus, contributing to the maintenance of cultures. Word problems, for example, have existed for thousands of years and their persistence contributes to the maintenance of some aspects of the culture of mathematics teaching down the ages. 
Finally, in this cultural function, genres embody ideological perspectives (Bakhtin 1986; Hanks 1987). Word problems, for example, are not only a method for organizing the application of mathematical techniques; they represent a particular set of assumptions about what it means to do and learn mathematics, assumptions about the situations depicted in the word problem, and assumptions about the students doing the word problem (Barwell 2018).

Applying these ideas to academic journals in mathematics education, we can quickly identify some common genres of text:

- Empirical research report

- Theoretical paper

- Book review

- Response or reaction to a previously published article

- Editorial

Many journals in mathematics education do not publish much outside this set of genres. The features of these genres can be deduced from examining multiple examples (see Table 19.1 for a summary). An empirical research report, for example, commonly includes the following:

- an introduction stating the problem

- a review of relevant literature

- a theoretical framework

- an account of research design and methods of data collection and analysis

- results

- discussion and conclusions

- references

Table 19.1 Summary of common components and functions of widely used genres of text appearing in mathematics education journals

\begin{tabular}{l|l|l}
\hline Genre & Common principal structural components & Function \\
\hline $\begin{array}{l}\text { Empirical research } \\
\text { report }\end{array}$ & $\begin{array}{l}\text { Literature review, theoretical framework, } \\
\text { research design and methods, results, } \\
\text { discussion and conclusions }\end{array}$ & $\begin{array}{l}\text { To report results of } \\
\text { research }\end{array}$ \\
\hline Theoretical paper & $\begin{array}{l}\text { Review of theoretical literature, } \\
\text { presentation of new theoretical } \\
\text { proposition, illustration of theoretical } \\
\text { proposition }\end{array}$ & $\begin{array}{l}\text { To propose new } \\
\text { theories or theoretical } \\
\text { developments }\end{array}$ \\
\hline Book review & $\begin{array}{l}\text { Summary of a book, discussion of merits } \\
\text { and contribution of book }\end{array}$ & $\begin{array}{l}\text { To discuss new } \\
\text { book-length } \\
\text { contributions to the } \\
\text { field }\end{array}$ \\
\hline $\begin{array}{l}\text { Response or reaction to } \\
\text { a previously published } \\
\text { article }\end{array}$ & $\begin{array}{l}\text { Summary of selected points from } \\
\text { previously published article, discussion or } \\
\text { critique of these points }\end{array}$ & $\begin{array}{l}\text { To contribute to } \\
\text { academic debate }\end{array}$ \\
\hline Editorial & $\begin{array}{l}\text { Comments on a specific topic of relevance } \\
\text { to readers }\end{array}$ & $\begin{array}{l}\text { To orient readers to } \\
\text { topics of interest in the } \\
\text { journal }\end{array}$ \\
\hline
\end{tabular}


These components have important functions. Indeed, a key part of academic socialization in mathematics education is about using and understanding these components (the structure is similar for other texts, including doctoral theses or PME papers). The structure serves to organize the various components of a research report in a way that, to a well-socialized member of the academic community of mathematics education, is recognizable and hence easy to navigate, interpret and, significantly, evaluate. While there has been evolution over time, these basic features are well established and can be seen in papers published in the last 40 years. These genres of academic writing therefore pass on and maintain a particular culture of academic writing - a culture that is, by the way, relatively specific to mathematics education. Research reports in journals of chemistry, mathematics, or even applied linguistics all look a bit different from research reports in mathematics education journals. Moreover, these genres embed various ideological commitments, such as the assumption that the learning and teaching of mathematics can be understood through work that can be presented in the generic form of a research report. This assumption entails various deeper beliefs about learning and teaching mathematics as amenable to some kind of scientific, empirical process. Such texts also construct readers in a particular way - as being, for example, familiar with theory and research methodology, and the often complex technical language that might go with them.

Later in this chapter, we discuss the distinctive nature of article genres published in FLM. First, though, we need to provide some context.

\subsection{Historical Context}

In 1968 Hans Freudenthal launched a new journal, Educational Studies in Mathematics (ESM), devoted to research in mathematics education. He did so over objections from the secretary of the International Mathematics Union, who wondered if "there is a market for two international journals of that kind" (Furinghetti and Giacardi 2010, p. 33). The only other international journal in the field at that time was L'Enseignement Mathématique, first published in 1899, and the official organ of ICMI since ICMI's foundation (as the International Commission on the Teaching of Mathematics) in 1908. The field of mathematics education was clearly ready to support more journals, as ESM was joined in 1969 by Zentralblatt für Didaktik der Mathematik (ZDM), founded by Hans Georg Steiner and Heinz Kunle, and in 1970 by the NCTM's The Journal for Research in Mathematics Education (JRME). About a decade later Robert Davis's Journal of Children's Mathematical Behavior (JMB) joined the field, and shortly thereafter (in 1980) the first issue of Recherches en didactique des mathématiques (RDM) appeared. This was the context in which David Wheeler founded FLM, hoping that it would be different from the existing offerings. In the first issue in July 1980, he wrote as follows: 
My hope that this journal may grow into one that learns along with its writers and its readers is my justification for introducing a new journal into a crowded field. "Print, print, and still more print. Who needs it?", as several people have said to me, using other words. The dangers are worth risking, I think. A new journal makes no demands by itself: only people do that, on themselves or on others. And although life, including the life of classrooms, will no doubt go on much the same with or without For the Learning of Mathematics, well-chosen words can trigger awarenesses and stimulate reflections and give experience to those sensitive to them. If any who are reading this sigh at the prospect of yet more to read, I'd say they have missed the point. I want to do something to serve the interests of those who have to learn mathematics. I hope some who share that desire may find For the Learning of Mathematics a journal which it is in their own interest to read. (1980a, p. 2)

The title David Wheeler chose for his new journal reflected his desire to "do something to serve the interests of those who have to learn mathematics." In his editorial in the third issue of FLM he discussed the meaning of the title:

Being "for" the learning of mathematics in the sense of this journal's title means more than generalised approval. It means making an important issue out of the learning of mathematics, not taking it for granted, not remaining content with being ignorant about it. It means being aware and becoming aware of difficulties to study and to resolve. It means putting into circulation ideas and techniques that will eventually benefit those who want to learn mathematics. (1981, p. 2)

FLM is a journal for the learning, and the learners, of mathematics, rather than being of behavior or for didactics, research or teaching. The title echoes that of Gattegno's (1963) collection of articles, For the Teaching of Mathematics but with a very intentional shift in focus, from teaching and teachers to learning and learners. While most journals in mathematics education include the learning of mathematics as part of their scope (as an aspect of their focus on didactics, research or teaching), the title of FLM indicates a particular concern, not just with learning as a general category, but with the individual experience of learning mathematics.

\subsection{Aims of FLM}

The aims of FLM are stated on the inside front cover of each issue, and they have remained unchanged since the first issue appeared:

The journal aims to stimulate reflection on mathematics education at all levels, and promote study of its practices and its theories: to generate productive discussion; to encourage enquiry and research; to promote criticism and evaluation of ideas and procedures current in the field. It is intended for the mathematics educator who is aware that the learning and teaching of mathematics are complex enterprises about which much remains to be revealed and understood.

A few keywords stand out (at least in the minds of the editors): "stimulate reflection", "productive discussion", "encourage enquiry", "promote criticism". All of these suggest an opening out, rather than a drawing together of the discourse. 
What is sought are not answers, but new questions. There is more focus on what "remains to be revealed and understood" than on what is known and established.

Given these aims, the empirical research report is not a genre of writing that is often found in FLM. Research reports are often focused on reporting results, and unless those results are surprising enough to stimulate further reflection and inquiry, a research report is unlikely to fit the aims of FLM. Even a research report that does stimulate discussion might stimulate the same discussion more effectively if written in another way.

The aims of FLM were chosen by David Wheeler, and subsequent editors have been strongly guided by them. To understand FLM it is useful to return to David Wheeler's thinking about the journal, as reflected in his editorials in its first issues.

In issue 1(1) David Wheeler wrote:

An editor may want to ease into existence a journal with certain concerns, a certain style or tone, a certain level of discourse. (The inside front and back covers of this issue tell you something about what this editor has in mind. The contents of the issue tell you some more). (1980a, p. 2)

Over the years the "style and tone" of FLM, reflected already in its first issue, have been maintained, even as they have evolved. They reflect, perhaps, an FLM style of academic writing, which we hope in this article to describe.

As noted above, the inside front cover states the aims of the journal. The inside back cover offers "Suggestions to Writers" including a definition of "mathematics education":

"Mathematics education" should be interpreted to mean the whole field of human ideas and activities that affect, or could affect, the learning of mathematics. Articles about mathematics or about psychology, for example, are welcomed provided their content bears on the learning of mathematics: directly, or indirectly through offering a significant perspective to teachers of mathematics. The journal has space for articles which attempt to bring together ideas from several sources and show their relation to the theories or practices of mathematics education. It is a place where ideas may be tried out and presented for discussion.

This paragraph has evolved somewhat over the years, but its key point, that FLM, as an international journal of mathematics education, is open not only to articles that mathematics educators write, but also to articles that mathematics educators might want to read. The contents of that first issue reflect this idea, since it includes a graphic and commentary by the artist Josef Albers, reprinted from Despite Straight Lines (Bucher, 1977) a posthumous analysis of Albers' graphic constructions. Presumably this was selected by David Wheeler as an example of the kind of thing he wanted FLM to include. The next two issues include mathematical poems from the anthology Against Infinity (Robson and Wimp 1980), as well as an essay by Dick Tahta (1981) discussing the relation between mathematics and poetry, inspired by the anthology. Of course, there are also articles by mathematics educators about geometry, multiplication tables, word problems, and so on.

FLM aims to "generate productive discussion" and one sign of this is the inclusion of articles and communications that continue a discussion begun in a 
previous article. This genre of writing was encouraged by David Wheeler from the beginning. He wrote in the second issue:

I would like to see perhaps one fourth of the pages of each issue given over to correspondence and comments arising out of the articles that appear in the journal. Whether that measure of open discussion is achievable, I don't know. I cannot think of any journal which manages so much, but perhaps the others haven't tried. (1980b, p. 2)

It is rare that FLM manages to fill a fourth of its pages with such contributions, but it has happened. And perhaps more importantly, FLM is still trying. This means that the genre of comment finds a place in its pages. Comments need not only be inspired by articles that appear in the journal. FLM does not publish book reviews, ${ }^{1}$ but it does publish articles that are inspired by reading a particular book (like Dick Tahta's essay mentioned above).

\subsection{Genres of FLM Articles}

We have already mentioned commentaries on prior publications and poems as genres published in FLM. These are not the genres most often represented in its pages, however. Most FLM articles could be called essays, but other important genres include narratives and dialogues.

\subsubsection{Essay}

The word 'essay' comes from 'essayer' and an essay is a trying or testing of an idea. It can take many forms, but importantly, it should be about something. This can be a critical take on a common assumption, a reinterpretation of a well-known phenomenon, a questioning of theoretical assumptions, interrogation of a surprising event, reflection on the nature of learning, or many other topics. Whatever it is about, that idea should be tested, argued, questioned and interpreted in multiple ways. It should be put on trial.

In Reification as the birth of metaphor, in issue 14(1), Anna Sfard (1994) puts the concept of 'understanding' on trial. She begins with an interesting quote, states the topic of her essay in one sentence, and then relates a brief anecdote that sets the stage. She clearly says what she plans to do:

I soon discovered that, as far as the issue of understanding is concerned, current developments in the psychology of mathematics go hand-in-hand with some of the most significant recent advances in linguistics and in philosophy. [...] In this paper I will show how the idea of reification - the basic notion of the conceptual framework on which I have been working for quite a long time now-combines with the new general theories of

\footnotetext{
${ }^{1}$ Almost every rule of FLM has an exception. The exception to this rule appeared in issue 5(1).
} 
understanding. I hope to make it clear that the theory of reification is perfectly in tune with the latest philosophical and linguistic developments. (p. 44)

She spends some pages describing "the latest philosophical and linguistic developments", which is fine as we know how this will be relevant. She brings in some empirical results, but always in the service of her quest to explore 'understanding'. The quotes from the mathematicians she interviewed illustrate and elaborate the points she is making and are connected to the theory she has already introduced and other concepts as they become relevant. In the last page and a half she brings it together, showing, and illustrating with her data, how the theory of reification connects with the embodied metaphors of Lakoff and Johnson.

\subsubsection{Narrative}

A narrative tells a story. It should have a beginning, middle and end, and characters we care about. And like an essay it should be about something. David Reid's favourite example of this genre is Norman, by Jennifer MacPherson (1987), in issue 7(2).

It says right away what it is about: "the conceptual gulf that may exist between cultures [...] which may allow a teacher to [...] pursue goals that are conceptually incompatible with those of her students" (p. 24). That is a topic that should interest us all. And then the story starts. The stage is set. The context is described in no more detail than we need.

The writing is engaging. More academic articles should have a paragraph, on the first page, that begins "The guinea pigs did not flourish". In the next paragraph we meet the main character, Norman. He is building a sled out of Lego. Then the story takes a surprising turn, and the writing becomes more detailed, giving us the 'data' we will need for the later 'analysis'.

The narrative part of the essay is now over, and the discussion continues, ranging over topics such as language, gender, the relationships between humans and non-human animals, mathematical competencies, cultural relevance, and the goals of education. All this in less than 2500 words, without any (explicit) references.

\subsubsection{Dialogue}

The closing article in the FLM special issue on ethnomathematics ${ }^{2}$ by Marcia Ascher and Ubiratan D'Ambrosio (1994) is a dialogue. In this case they worked from a recording of an actual conversation, but dialogues can also be constructed from exchanges of emails, for example, or out of whole cloth. The aim remains, however to reflect the thinking of two (or more) distinct individuals on an issue or

\footnotetext{
${ }^{2}$ FLM does not publish special issues. This is one of the exceptions.
} 
topic. Dialogues have a deceptively simple structure: two or more authors have named turns, so that each writes in their own voice and with their own ideas. Unlike other forms of co-authorship, in dialogues different points of view are explicitly set against each other and explored, often without coming to any concluding consensus. This genre has appeared through FLM's history, starting with the first issue, in which the founding editor, David Wheeler, discusses the film "The Foundations of Geometry" with its creator, Caleb Gattegno (Gattegno and Wheeler 1980).

\subsubsection{Comment}

We have already referred to Wheeler's desire that the pages of FLM should include comments on previously published articles and such comments have regularly appeared, usually in a section of shorter pieces collectively labelled "Communications". Comments are a bit like the letters to the editor that appear in newspapers: the main requirement for a comment is that it is prompted by a previously (and usually recently) published article, which is mentioned in the first line. Comments may offer critique, or seek to extend or refute the ideas in the original article. Or not - comments may simply report interesting reflections prompted by the original article, but that may be considered tangential to its original theme.

\subsubsection{Other}

Taxonomists in biology know that it is vital not only to characterize the prototype of a species, but also to explore its variations, to map out the extent to which members of a species can look and behave differently but still interact is ways that marks them as parts of a whole. The genres of essay, narrative and dialogue are prototypical genres of FLM articles, but FLM articles can take on many forms while still being part of the FLM 'conversation'. For example, issue 34(1) includes what could be called a 'graphic article' akin to graphic novels in which drawings are used to tell a story. Issue 37(3) includes a work of fiction, a short story relating a mathematical exploration, while an article in issue 29(2) includes a fictional Socratic dialogue featuring Xanthippe, the wife of Socrates, and a slave girl named Menousa, that the authors wrote, by their own admission, "for fun" (Mason and Watson 2009). We leave it to readers to explore the pages of FLM to identify the many other minor and sometimes quirky genres.

\subsubsection{Some Comments on These Genres}

The genres we have described make FLM what it is and reflect its aims and values. While each of the genres is distinctive, there are some commonalities. They are 
rather looser than some commonly used genres in mathematics education journals. While there is structure, authors have quite a bit of scope to shape the writing (although within strict word limits). Another common feature, often implicit, is the place of authors' voices. Dialogues explicitly feature the voices of the authors, as do comments, but voice is also a feature of the main essay-form articles. The engaging opening of FLM articles is also a way to give the sense of a writing author, using first-person verb forms: "I argue" rather than "It is argued". The presence of authors' voices is related to the what might be called the ideology, or perhaps better the ethos of FLM, as reflected in the nature of the genres of article it includes, and which make it the distinctive journal that it is. FLM can be understood in terms of conversation: articles speak to each other and contribute to longer conversations that have developed in the pages of the journal over the years. Why 'conversation'? Because conversation reflects the view that that academic thinking is a collective activity, that ideas arise in response to other ideas, and that learning mathematics is a "complex enterprise" about which it is difficult to say anything with any finality. As Lesley Lee (2014) wrote in issue 34(1): "The conversation is not for those who feel they have all the answers but for those who are searching and willing to contribute their understandings and questions to the ongoing inquiry" (p. 6). Conversations do not end; there is always more to be said.

This spirit of conversational enquiry is embodied in the genres of FLM, and perhaps stands in contrast to some of the genres that do not appear in its pages. Research reports, for example, are designed to, well, report: they provide a self-contained account of a piece of research and, as such, do not invite a response. Of course, many journals sometimes include responses to research reports, and we don't claim that FLM has a monopoly on conversational style. But we do claim that FLM is a rare example of a mathematics education journal that is organized around an ethos of conversation.

For the rest of this chapter, we provide some information about the submission and review process.

\subsection{The Submission Process}

FLM does not use any management software to handle the submission process. Submissions are sent electronically to editor@flm-journal.org, or, if that is impossible, printed submissions can be sent by post (email the editor for the address). There are no formatting requirements for initial submissions. Articles should generally be within the range of 2500-5000 words. Longer articles are rarely accepted, and are then published in two or more parts. Short communications (for example, comments on already-published articles) should generally contain fewer than 2000 words.

Contributions may be submitted in English or French. The English may be US, British, or some other self-consistent variant. FLM is an international journal and welcomes submissions from all parts of the world. The editors recognize that the 
languages of publication give an advantage to authors who are native speakers of French and English. Encouragement and support is given to authors who are not writing in their first language.

Authors are strongly advised to read the "Suggestions to Writers" found on the inside back cover of each issue, and on the website flm-journal.org. The last line of these suggestions reads "Current house style may be inferred from the articles in $\mathbf{3 8}$ (1) and later issues." This should be taken as an invitation to become familiar with recent articles, but there is no expectation that initial submissions conform perfectly to our house style. Accepted manuscripts are carefully edited by the editor prior to publication.

If submissions are submitted blind, then they are reviewed blind; otherwise reviewers know the identities of authors. In other words, authors may choose to have a blind review or not, according to what they submit. Similarly, reviewers may choose to identify themselves in their reviews, and if they do not then blind reviews are sent to authors.

For the Learning of Mathematics is edited by the Editor and two Associate Editors, supported by an Advisory Board of about two dozen mathematics educators, all of whom have previously published in FLM. The first stage of our review process is an internal reading by the editors and members of the Advisory Board. This first stage is usually quick and is intended to sort out submissions that are not well suited to FLM from those that are. Many submissions are rejected at this first stage, usually because they are research reports, teaching activities, or mathematical results that are better published elsewhere. If an issue, problem or observation in such a submission could form the focus of an essay or narrative, authors are may be encouraged to submit a new piece of writing in a more suitable genre. Suitable submissions may receive feedback as to revisions that should be done prior to external review, to allow that review to be more productive. Suitable submissions are sent to two or more external reviewers, who are experts in the field, and familiar with the journal, its style and aims. It is normal that the external review leads to several cycles of revisions before manuscripts can be published, although an indication of provisional acceptance is often made early in this process.

The review process requires at least two months and usually rather longer. Shorter communications may be handled more quickly. FLM is published three times a year, in March, July and November, and the process of editing, typesetting and printing each issue takes about four months, so articles accepted in March appear in July.

\subsection{Some Advice}

A feature of the different genres of articles that appear in FLM is that they often have engaging openings. Most readers are reading out of interest, not obligation, and so it is important that the beginning of an article be interesting. This is especially important because a common strategy for deciding if an article is worth 
reading, reading the abstract, involves a trip to the web site, as the abstracts of articles appear only there. Instead, the title of an FLM article and the first few paragraphs must engage the reader's attention.

There are almost as many ways to engage the reader as there are FLM articles. Some start with a provocative quote. Some start with an interesting bit of transcript or a narrative of something interesting that happened in a classroom. Some start by asking a question. We encourage you to pick an issue at random and to look through it for an article that piques your interest, on a topic that is not a research focus of yours. Look at the beginning. How did the authors engage your attention?

Once readers are interested, their interest has to be maintained. Not every paragraph has to be as fascinating as the first one, but it is important not to try the readers' patience too much. One thing to avoid is 'academic throat clearing'. ${ }^{3}$ This is the listing of other researchers who consider your topic worth discussing, definitions of terms you will later use a few times, and so on. For some reason, academic writing often includes such material towards the beginning, before finally getting to the point some paragraphs (or pages) later. One feedback we often give authors is along the lines of 'delete everything on pages 2-5'. As with giving a speech, do your throat clearing in private, and then start talking when you are ready.

For the reader, it is helpful to know what an article is about quite quickly and without making much of an effort. It is tempting to spring a surprise, to bring something unexpected in at the end, but the danger is that no one reads to the end, and so no one is surprised. As editors, we read many articles out of obligation, not out of interest, so we stick it out to the end, and sometimes find ourselves pleasantly surprised. If that happens then the advice is always to at least hint strongly at the surprise early on.

So, we've said something about the kind of writing that appears in FLM. If you have questions, we urge you to read the journal: back issues are available without subscription at flm-journal.org. If you have an idea for a contribution, you can contact the editor to discuss it at editor@flm-journal.org.

To conclude, we return to the words of the founding editor. In an editorial in the last issue he edited, 17(2) David Wheeler (1997) expressed his gratitude to:

authors with something germane, intriguing, and substantive to say-something that provokes thought and brings a fresh point of view-and who write in ways that take account of their readers, speaking plainly and putting their cards on the table. (p. 2)

This sums up our advice to prospective authors: have something thought provoking to say, and say it as clearly as you can.

\footnotetext{
${ }^{3}$ This phrase has been used by FLM editors for some time. We learned it from Laurinda Brown who learned it from Dick Tahta. We assume David Wheeler was familiar with the concept, and probably also with the phrase, which pops up from time to time in critiques of academic writing.
} 


\section{References}

Ascher, M., \& D’Ambrosio, U. (1994). Ethnomathematics: A dialogue. For the Learning of Mathematics, 14(2), 36-43.

Bakhtin, M. M. (1986). Speech genres and other late essays (C. Emerson \& M. Holquist, Eds., W. McGee, Trans.). Austin, TX: University of Texas Press.

Barwell, R. (2018). Word problems as social texts. In K. Yasakuwa, A. Rogers, K. Jackson, \& B. V. Street (Eds.), Numeracy as social practice: Global and local perspectives (pp. 101-120). Abingdon, UK: Routledge.

Bucher, F. (1977). Josef Albers: Despite straight lines: An analysis of his graphic constructions. Cambridge, MA: MIT Press.

Furinghetti, F., \& Giacardi, L. (2010). People, events, and documents of ICMI's first century. Actes d'Història de la Ciència i de la Tècnica, Nova Època, 3(2), 11-50.

Gattegno, C. (1963). For the teaching of mathematics (Vol. 1). Reading, UK: Education Explorers.

Gattegno, C., \& Wheeler, D. (1980). The foundations of geometry. For the Learning of Mathematics, 1(1), 10-16.

Gerofsky, S. (1996). A linguistic and narrative view of word problems in mathematics education. For the Learning of Mathematics, 16(2), 36-45.

Hanks, W. F. (1987). Discourse genres in a theory of practice. American Ethnologist, 14(4), 668-692.

Lee, L. (2014). The FLM conversation. For the Learning of Mathematics, 34(1), 6-7.

Macpherson, J. (1987). Norman. For the Learning of Mathematics, 7(2), 24-26.

Mason, J., \& Watson, A. (2009). The Menousa. For the Learning of Mathematics, 29(2), 33-38.

Robson, E., \& Wimp, J. (1980). Against infinity: An anthology of contemporary mathematical poetry. Parker Ford, PA: Primary Press.

Sfard, A. (1994). Reification as the birth of metaphor. For the Learning of Mathematics, 14(1), 44-55.

Swales, J. M. (1990). Genre analysis: English in academic and research settings. Cambridge, UK: Cambridge University Press.

Tahta, D. (1981). On poetry and mathematics. For the Learning of Mathematics, 1(3), 43-47.

Wheeler, D. (1980a). Editorial. For the Learning of Mathematics, 1(1), 2.

Wheeler, D. (1980b). Editorial. For the Learning of Mathematics, 1(2), 2.

Wheeler, D. (1981). Editorial. For the Learning of Mathematics, 1(3), 2.

Wheeler, D. (1997). Editorial. For the Learning of Mathematics, 17(2), 2.

Open Access This chapter is licensed under the terms of the Creative Commons Attribution 4.0 International License (http://creativecommons.org/licenses/by/4.0/), which permits use, sharing, adaptation, distribution and reproduction in any medium or format, as long as you give appropriate credit to the original author(s) and the source, provide a link to the Creative Commons license and indicate if changes were made.

The images or other third party material in this chapter are included in the chapter's Creative Commons license, unless indicated otherwise in a credit line to the material. If material is not included in the chapter's Creative Commons license and your intended use is not permitted by statutory regulation or exceeds the permitted use, you will need to obtain permission directly from the copyright holder. 